Attention Tracker, presented as percentile ranks using normative data built-into the ImPACT QT.

Results Zambian athletes scored nearly two standard deviations below the mean on Motor Speed (7th percentile), when compared to North American normative data. However, performance on Attention Tracker (44th percentile) and Memory (56th percentile) among Zambian athletes was well within the average range.

Conclusions This data is the first to explore Zambian athletes' performance on any concussion measure. Zambian athletes performed poorly on the ImPACT QT factor score related to motor speed. These results suggest that Zambian athletes may be unfamilar with testing on an iPad device or there may have been cultural barriers in the interpretation of test directions. Further, understanding these preliminary performance measures are a starting point that could aid in understanding which concussion measures would be culturally appropriate to implement in the prevention, diagnosis, and care of concussion among Zambian athletes.

\section{NORMATIVE BASELINE SCAT5 SCORES IN A POPULATION OF UNITED STATES PARALYMPIC ATHLETES}

1,5Laura Zdziarski, 2,5Lauren Pierpoint, 3,5David Taylor, ${ }^{3,5}$ Amber Donaldson, ${ }^{4}$ William Moreau, ${ }^{3,5}$ Dustin Nabhan. 'University of Utah, Department of Orthopaedics, Salt Lake City, USA; ${ }^{2}$ Steadman Philippon Research Institute, Vail, USA; ${ }^{3}$ USA Olympic and Paralympic Committee, Colorado Springs, USA; ${ }^{4}$ Southern California University of Health Sciences, Whittier, USA; ${ }^{5}$ USA Coalition for the Prevention of Illness and Injury in Sport, Colorado Springs, USA

\subsection{6/bjsports-2021-IOC. 154}

Background The Sport Concussion Assessment Tool $5^{\text {th }}$ Edition (SCAT5) is the most recent version of the concussion evaluation tool used by clinicians to evaluate athletes with suspected concussions.

Objective To describe normative baseline SCAT5 scores among United States Paralympic athletes.

Design Retrospective descriptive epidemiology.

Setting United States Olympic and Paralympic Sports Medicine Centers.

Participants Fifty-eight Paralympic athletes $(60.3 \%$ female, mean age \pm standard deviation $(S D)=30.2 \pm 10.6)$ representing 7 sport federations underwent baseline SCAT5 testing between April 2018 and July 2019.

Interventions None.

Main Outcome Measurements Baseline SCAT5 scores of healthy Paralympic athletes. T-tests were used to compare scores by sex.

Results Athletes reported an average of 5.0 5.4 symptoms (median $=2.5, \mathrm{IQR}=1.0-8.5)$ with an average severity score of $11.0 \pm 14.3$ (median=6.0, IQR=0-17.5). Over half of athletes $(59.3 \%)$ reported having sustained at least one concussion prior to testing (range $=0-16$ reported concussions). Mean scores \pm SD for major components of the SCAT5: $4.8 \pm 0.74$ for orientation, $21.0 \pm 3.7$ for immediate memory, $3.9 \pm 1.1$ for concentration, $6.7 \pm 6.4$ for balance, $6.9 \pm 2.2$ for delayed recall, and $15.7 \pm 2.8$ for Standardized Assessment of Concussion. No sex differences were observed for any component scores of the SCAT5.

Conclusions Normative values for baseline SCAT5 performance are presented for a population of healthy Paralympic athletes. Future research should focus on the development of normative data for specific Paralympic disability classifications.
Clinical relevance Knowledge of normative scores may aid clinicians' interpretation of baseline and post-injury SCAT5 scores.

\section{THE TOP 1\% OF HEAD IMPACTS CAUSE VISIBLE SIGNS AND 'CHECK ENGINE' RESPONSES IN ATHLETES AND MILITARY SERVICE MEMBERS}

Adam Bartsch. Prevent Biometrics, Minneapolis, USA

\subsection{6/bjsports-2021-IOC.155}

Background For the past $\sim 20$ years, the scientific literature, based on data from inaccurate helmet/head-mounted sensors, has reported that there is little link between head impact magnitude and location and visible signs (VS). The current study found that VS were seen only in impacts in the top $1 \%$ as measured by head impact acceleration, velocity and/or energy. Objective Head impacts were measured with an accurate head impact monitoring mouthguard and confirmed through video and data trace analysis. The athlete/military service member behavior was observed for a visible sign post-impact.

Design Retrospective meta-analysis from military parachute, boxing, mixed martial arts, weapons firing, as well as American football, Boxing, Ice Hockey, Karate, Lacrosse, Mixed Martial Arts, Rugby, Tae Kwon Do, Football.

Setting Sporting field or Military training activity.

Participants 50,000 verified impacts over 10,000 person-days. Assessment of Risk Factors Visible signs post-impact.

Main Outcome Measurements Kinetic energy transfer (KE), peak scalar linear acceleration (PLA), peak scalar linear velocity (PLV), impact location, impact direction, type of VS.

Results There were fifty (50) single head impacts that caused visible signs. The most common visible signs were loss of consciousness, posturing, dazed, imbalance and ataxia. All visible signs impacts were in the top $1 \%$ by magnitude $(40-100 \mathrm{~g}, 3-$ $8 \mathrm{~m} / \mathrm{s}$ and $30-150 \mathrm{~J}$ ). A total of $90 \%$ of the VS impacts were to the side or rear of the head.

Conclusions Visible signs do not occur often, but always occurred in the top $1 \%$ of head impacts by magnitude, and $90 \%$ of these were to the rear and the sides of the head. By monitoring head impacts with an accurate device, clinical staff can have access to data that identifies head impacts most likely to cause visible signs.

\section{RISK FACTORS FOR POST-CONCUSSION SUBSEQUENT MUSCULOSKELETAL INJURIES}

${ }^{1}$ Thomas Buckley, ${ }^{3}$ Jessie Oldham, ${ }^{1}$ Caroline Howard, ${ }^{2}$ Robert Lynall, ${ }^{1}$ Buz Swanik ${ }^{1}$ Nancy Getchell. 'University of Delaware, Newark, USA; ' University of Georgia, Athens, GA, USA; ${ }^{3}$ Boston Children's Hospital, Waltham, MA., USA

\subsection{6/bjsports-2021-IOC.156}

Background Elevated rates (1.5 to 3.0 times) of musculoskeletal (MSK) injuries in the first year post-concussion have been recently identified in diverse athletic populations; however, clinically feasible risk factors have received limited attention.

Objective To identify clinical predictors of post-concussion subsequent musculoskeletal (MSK) injuries.

Design Prospective longitudinal.

Setting U.S. Intercollegiate Athletics . 